\section{Efectos de un programa de mejoramiento de la calidad en servicios materno perinatales en el Peru: la experiencia del proyecto $\mathbf{2 0 0 0}$}

\section{Effects of a continuous quality improvement program in maternal and perinatal health services in Peru: the experience of Project 2000}

Juan A. Seclen-Palacín 1

Enrique Jacoby $\mathrm{M} 2$

Bruno Benavides $C_{3}$ Joaquín Novara $\mathrm{V} 4$

Anibal Velásquez $\vee 5$

Enrique Watanabe $\mathrm{V} 6$

César Arroyo V 7

1 Organización Panamericana de la Salud. Representación Brasil. Setor Embaixada Norte, Lote 19. Brasilia, DF, Brasil. CEP: 70.800-400. E-mail: seclen@bra.ops-oms.org

2 Area de Medicina Familiar y Comuinitaria. Unidad de Nutrición,

Organización Panamericana de la Salud. Washington, DC, Estados Unidos 3 Oficial de Programa para la Región de América Latina. Partners in Population and Development. Dhaka, Bangladesh

4 Departamento de Estadística Universidad Peruana Cayetano Heredia. Lima, Perú

5 Departamento de Medicina Preventiva. Universidad Nacional Mayor de San Marcos, Lima. Perú

6,7 Consultor en Salud Pública. Lima, Perú

\section{Abstract}

Objectives: to compare the quality of the maternal-perinatal health services (MPHS) between health facilities with a continuos quality improvement (CQI) and those without such program; to evaluate the knowledge and attitudes of the users of maternal health services; to determine the contribution of the $C Q I$ on the level of quality.

Methods: it was conducted a CQI program in 74 selected health facilities from Perú (1996-2000) for improved the quality of MPHS. A controlled, quasiexperimental study in facilities health (hospital, health centers and peripheral facilities) was conducted. The quality of care was measured by scores of the MPHS by comparing averages; the knowledge and attitudes of users by bivariate analysis, and the prediction of the quality level was studied through multivariate analysis.

Results: at the end of the intervention, CQI facilities score was 61,8 points and of 37,5 in the comparison group, $(p<0,001)$. The average of obstetrical warms signs knowledge was greater in CQI facilities (3,6 signs/interviewed) versus 2,5 in comparison facilities users, $(p<0,05)$. The intention of institutional childbirth was two times greater among users of CQI facilities, $(p=0,038)$. The main predictions factors of quality were the CQI intervention and the availability of health professionals.

Conclusions: the level quality is greater in the participant health facilities (with CQI). The users of these facilities had more knowledge of warning signs and better attitude towards the institutional delivery. The CQI has contributed to those achievements.

Key words Quality assurance, health care, Evaluation, Maternal health services, Maternal welfare

\section{Resumo}

Objetivos: comparar la calidad de los servicios de salud materno perinatales (SMP) de los establecimientos participantes en un programa de mejora de calidad (PMC) y compararlos con un grupo control; evaluar comparativamente los conocimientos y actitudes de contribución del PMC en los niveles de calidad.

Métodos: fue implementado un PMC en 74 establecimientos de salud seleccionados para mejorar la calidad. Se desarrolló un cuasiexperimento controlado en 74 hospitales, centros de salud y establecimientos periféricos. El análisis de la calidad de los SMP se realizó mediante comparación de promedios de escalas centesimales; los conocimientos y actitudes de las del nivel de calidad a través de análisis multivariado.

Resultados: al final de la intervención, la puntuación de los establecimientos con PMC tuvo un promedio de 61,8 puntos y de 37,5 en el grupo de comparación, $(p<0,001)$. El promedio de conocimientos de signos de alarma obstétricos fue mayor en las usuarias $P M C$ (3,6 signos/entrevistada) versus 2,5 en usuarias sin PMC, $(p<0,05)$. La intención de parto institucional fue dos veces mayor en las usuarias de los servicios con PMC, $(p=0,038)$. Los predictores significativos de calidad fueron la mejora continua de la calidad y la disponibilidad de profesionales de salud.

Conclusiones: el nivel de calidad es mayor en los servicios que desarrollaron PMC. Las usuarias de tales servicios tienen más conocimientos de los signos de alarma obstétricos y mejor actitud hacia el parto institucional. El PMC ha contribuido para tales logros.

Palavras-chave Garantia de la calidad de Atencion de Salud, Evaluación, Servicios de salud materna, Bienestar materno usuarias de servicios de salud materna; y determinar la usuarias mediante análisis bivariado; y la predicción 


\section{Introduccion}

La mortalidad materna es un indicador sensible del estado de la mujer en la sociedad, refleja el acceso a los servicios de salud y la calidad de atención recibida. ${ }^{1}$ En el año 1987, durante la Reunión Internacional de Maternidad Saludable y Segura realizada en Nairobi (Kenya) se estableció como meta para los países en vías de desarrollo la reducción de la mortalidad materna en un 50\% hacia el año 2000,2,3 recientemente las metas de milenio (Naciones Unidas, 2000) insta a los gobiernos a reducir la mortalidad materna en un 75\% hacia el 2015. En América Latina y el Caribe, debido a los elevados índices de mortalidad materna, los gobiernos y ministerios de salud 4 desarrollaron esfuerzos por mejorar la atención de la salud de la gestante; 5 para ello se implementaron diversas estrategias. ${ }^{6} \mathrm{La}$ promoción del control prenatal, las intervenciones sobre nutrición en gestantes, el enfoque de riesgo obstétrico, la promoción del parto institucional y del parto limpio en domicilio atendidos por parteras tradicionales, la oportuna accesibilidad a establecimientos que brinden funciones obstétricas esenciales de calidad, entre otras, fueron las estrategias de mayor difusión y aplicación.7-10 Adicionalmente, se están haciendo esfuerzos por identificar y extender las estrategias más costo-efectivas para países en vias de desarrollo,5,11 para lo cual se vienen realizando estudios de evaluación de resultados e impacto de intervenciones en salud materna. ${ }^{12,13}$

El Perú es uno de los países con mayor mortalidad materna en las Américas; 14 en el año 1996 ocurrieron 265 muertes maternas por cada 100.000 nacidos vivos. 15 Desde entonces se han dedicado mayores esfuerzos y recursos para lograr un incremento en las coberturas de atención del control prenatal, parto institucional y acceso a la atención de calidad ante complicaciones obstétricas y perinatales; 16,17 uno de tales esfuerzos es el Proyecto 2000 (P2000), el cual surgió de la cooperación entre el Gobierno del Perú y la Agencia de los Estados Unidos para el Desarrollo Internacional (USAID), con el propósito de contribuir en la reducción de la mortalidad materna en el Perú. Desde finales de 1996, el Proyecto implementó un programa de mejoramiento continuo de la calidad de atención (PMC) en los servicios materno perinatales (SMP) de 89 Hospitales y Centros de Salud ubicados en doce departamentos. El enfoque conceptual y metodológico del modelo de calidad del Proyecto 2000 se basa en la propuesta de Bruce 18 para los servicios de planificación familiar. La conducción de procesos de mejora continua de la calidad en los servicios, articulados a las caracterís- ticas culturales y expectativas de la población (demanda), con la intención de proporcionar SMP accesibles, aceptables y de calidad, 19 constituyeron el eje de acción del Modelo del P2000.

Luego de cinco años de actuación y con fines de conocer los efectos del PMC se realizó el presente estudio, siendo sus objetivos: compara la calidad de atención de los SMP de los establecimientos participantes del programa y compararlos con un grupo de establecimientos control; evaluar comparativamente los conocimientos y actitudes de usuarias de servicios de salud materna y, determinar la contribución del PMC en los niveles de calidad.

\section{Metodos}

\section{Ámbito de intervención}

El Perú es un país ubicado en la zona central y occidental de Sudamérica. Tiene una población aproximada de 26 millones de habitantes y alrededor de 700.000 mujeres embarazadas, 20 dicho país se caracteriza por su diversidad geográfica, climatológica, étnica, económica, social y cultural. En sus tres regiones geográficas, costa, sierra y selva, existen poblaciones rurales con limitada accesibilidad a los servicios sociales del estado, incluidos los de salud. Se estima que una de cada dos familias peruanas se encuentra en condición de pobreza, existe un 11,5\% de mujeres sin educación, una de cada tres mujeres rurales tiene percepción de enfermedad, y sólo el $32,3 \%$ de ellas realiza alguna consulta de salud.21 Un $67 \%$ de gestantes del Perú tuvo al menos una atención prenatal institucional, el 49,6\% tuvo parto en servicios de salud atendido por personal capacitado y, el 48,3\% de las embarazadas dio a luz en su domicilio, siendo este último valor mayor en la sierra $(72.2 \%)$ y selva $(65,1 \%) .15$

El Proyecto 2000 intervino en doce departamentos del Perú, los de mayores niveles de pobreza y menor accesibilidad a los servicios salud maternos, así en dos de ellos (Puno y Huancavelica) las coberturas de atención de parto en servicios de salud eran del $17,8 \%$ y $7,1 \%$, respectivamente. $15 \mathrm{La}$ intervención se dirigió a 18 Hospitales y 71 Centros de Salud.

\section{La intervención Proyecto 2000}

El Programa de mejora de la calidad de procesos involucró el desarrollo operacional, metodológico e instrumental de cinco componentes de la calidad: Uso de datos, Manejo estandarizado, Educación per- 
manente en servicios, Gestión y disponibilidad de recursos, y Relación proveedor-usuario-comunidad.

- Uso de datos, se refiere al proceso de recolección, procesamiento de datos y uso de información en decisiones gerenciales y clínicas del área materno perinatal.

- Manejo estandarizado se relaciona con la estandarización y protocolización de procedimientos médicos de atención obstétrica y neonatal.

- $\quad$ Educación permanente en servicios se basa en el análisis grupal y horizontal que el personal de salud realiza sobre la práctica diaria de los procesos de atención materno perinatal.

- Gestión y disponibilidad de recursos se orienta hacia la optimización de la gestión local para el suministro adecuado y oportuno de insumos, equipos y medicamentos básicos en la atención.

- Relación proveedor-usuario-comunidad destinado a favorecer el acceso precoz, oportuno y continuo de la gestante hacia los servicios obstétricos mediante estrategias efectivas de comunicación interpersonal y educación en salud; y la mejora de la cordialidad, el trato, respeto y empatía hacia las usuarias; así como también favorecer que los servicios, los agentes locales de salud y la comunidad desarrollen acciones conjuntas en pro de la salud de la mujer embarazada.

El modelo promovía el trabajo en equipo, las reuniones multidisciplinarias de análisis crítico de la práctica diaria, la toma de decisiones gerenciales basada en información, el seguimiento de usuarias, la incorporación de percepciones culturales de las mujeres en la oferta de servicios, estrategias de comunicación personal durante la consulta obstétrica, el trabajo comunitario, y la estandarización y revisión continua de los procedimientos clínicos y quirúrgicos obstétricos. Los Hospitales y los Centros de Salud se constituyeron además en sedes locales de capacitación en salud materno infantil para profesionales y técnicos de salud del nivel periférico. En los establecimientos periféricos (centros de salud pequeños y puestos de salud urbano marginales y rurales), la intervención del proyecto fue indirecta, ya que los profesionales de estos establecimientos acudían a las sedes locales de capacitación para recibir entrenamiento en servicio sobre mejoramiento continuo de la calidad. Una vez concluido tal proceso, retornaban a sus establecimientos de origen con la misión de conducir la implementación del modelo en el nivel local. El modelo consideró que el desarrollo de los procesos de mejora de la calidad, deben tener efectos en dos niveles: 1) En el establecimiento de salud se debe disponer de servicios materno perinatales que brinden funciones obstétricas y neonatales esenciales de calidad y lograr una mayor aceptabilidad y accesibilidad de parte de la población, y 2) En la demanda se debe lograr una mayor utilización de los servicios obstétricos y neonatales, mayor protección de la salud de la gestante frente a complicaciones obstétricas y/o neonatales, y mayores niveles de conocimientos y prácticas de autocuidado en salud obstétrica y perinatal. A través de estos efectos se contribuiría en alcanzar un mejor estado de salud materna (Figura 1). 


\section{Figura 1}

Modelo de Intervención del programa de mejora continua de la calidad (MCC) del Proyecto 2000 y sus efectos

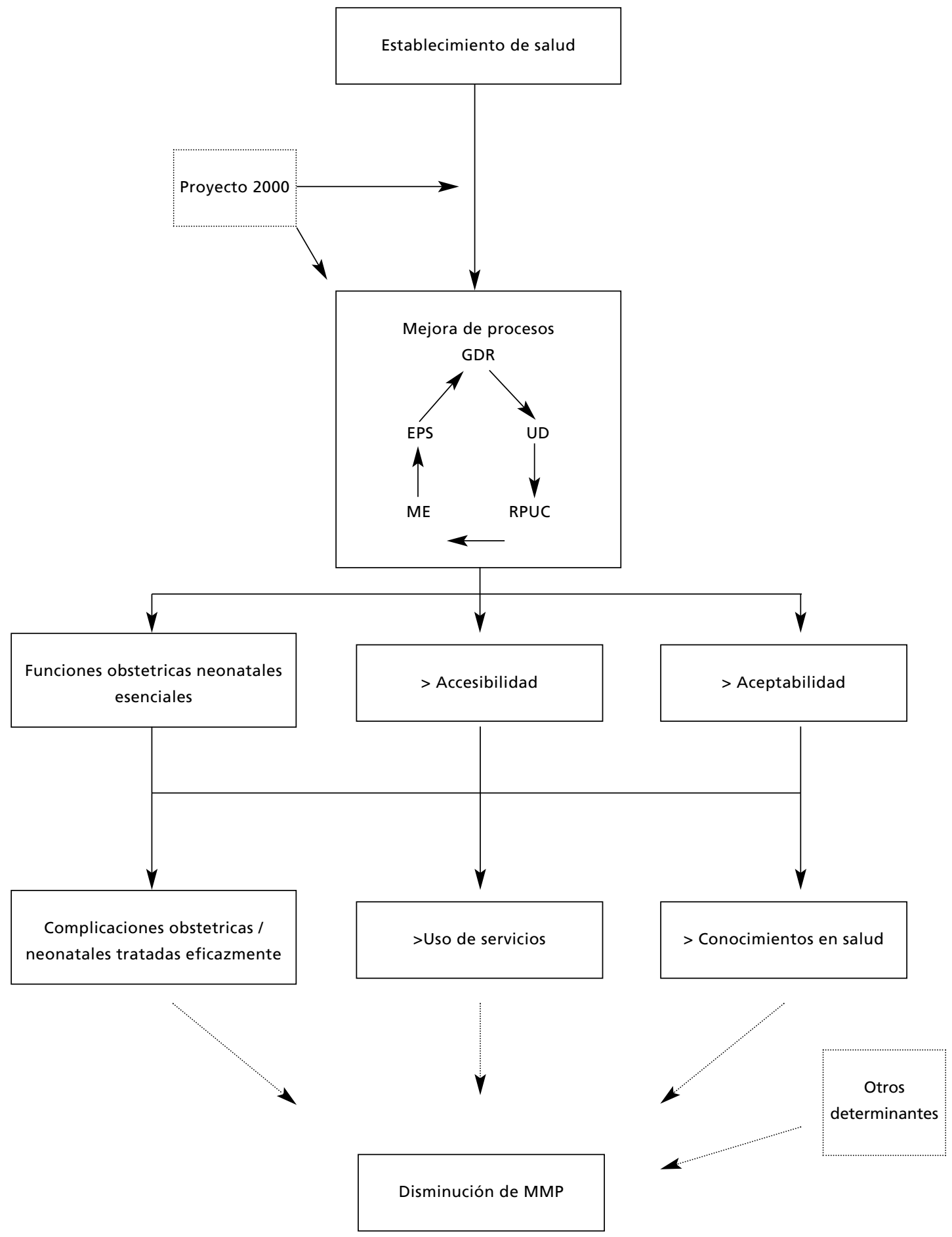

GDR = Gestión y disponibilidad de recursos; UD = Uso de datos; RPUC = Relación proveedor usuario comunidad; ME = Manejo estandarizado; EPS = Educación permanente en servicio; MMP = Mortalidad materna perinatal. 


\section{El estudio de campo}

Se realizó un estudio cuasiexperimental, con dos grupos de estudio, uno conformado por establecimientos donde intervino el Proyecto 2000 (Grupo P2000, de intervención) y aquellos donde no intervino (Grupo No P2000, control). El número de establecimientos necesarios para arribar a conclusiones probabilísticas se definió por la asociación (correlación) entre las variables nivel de calidad y grupo de estudio. Al hipotetizar una asociación de $\mathrm{r}=0,3$ (que equivale a que un $9 \%$ de la varianza), fueron necesarios incluir 68 establecimientos para detectar ese nivel de asociación (o superiores) con un Poder de $80 \%$ y una significancia de 5\%. Estos cálculos están basados en las tablas de poder muestral. Con fines de evitar pérdidas de datos ante contingencias del trabajo de campo, se incluyeron seis establecimientos adicionales haciendo un total de 74, distribuidos equitativamente en ambos grupos de estudio. El muestreo utilizado fue multietápico (estratificado, aleatorio simple, pareado). El proceso de muestreo tuvo varios momentos, en primer término, considerando las variables nivel de pobreza, tasa de analfabetismo, cobertura de partos institucionales, prevalencia de uso de anticonceptivos y tasa de mortalidad infantil se estratificaron los 34 departamentos de salud en tres niveles de desarrollo: alto, medio y bajo. Luego de ello, se seleccionaron aleatoriamente seis DISAs (Direcciones regionales de salud) por cada grupo de estudio incluyendo dos de cada estrato, así en el Grupo P2000 se incluyeron las DISAs de La Libertad, Ancash, Ucayali, Andahuaylas, Ica y Ayacucho, mientras que en el Grupo de comparación participaron Piura, Amazonas, Madre de Dios, Pasco, Lima Sur y Cusco. Posteriormente, de cada DISA se eligió aleatoriamente 1-2 Hospitales, 2-4 Centros de Salud, y 1 establecimiento periférico a fin de completar los 37 establecimientos requeridos para cada grupo. Al final se incluyeron en el estudio 23 Hospitales (11 del grupo P200), 33 Centros de salud (17 del P2000) y 18 Establecimientos periféricos (9 del P2000).

Las variables principales del estudio fueron: 1) Calidad de atención, definida como la agregación de los cinco componentes de la calidad, medida en escala continua y resultante de la sumatoria de los puntajes parciales de cada componente; 2) Conocimientos de las usuarias, definida como el grado de conocimientos de los signos de alarma obstétricos en usuarias de los servicios de atención prenatal, considerada como variable categórica y 3) Actitud de la usuaria, definida como la intención espontánea del uso de servicios de atención de parto institucional en usuarias de control prenatal, tipificada como variable categórica.

Se utilizaron dos instrumentos semi-estruturados de medición de la calidad (validados previamente en un ensayo piloto), una para los SMP de Hospitales y Centros de salud, y otra para establecimientos periféricos. La medición se hizo a través de entrevista, observación directa, listas de chequeo y revisión de archivos; los instrumentos contenian secciones y preguntas relacionadas con las variables del estudio. La recolección de datos fue realizada, durante los meses de Setiembre - Octubre del año 2000, por profesionales de salud (gineco-obstetras, pediatras, epidemiólogos y médicos especialistas en salud pública) rigurosamente entrenados. Calidad de atención fue investigada a través de la revisión de fuentes secundarias documentales (registros diarios de atención, registros de producción de servicios mensuales, historias clínicas de hospitalización, consulta externa y atención de emergencia, actas de las reuniones de calidad) y, entrevistas directas a los proveedores de salud. Conocimientos y actitudes en usuarias se midió a través de encuestas de salida a gestantes que acudieron al control prenatal. Además de ello, para determinar la contribución del Proyecto sobre el nivel de calidad, se recolectó información de factores que podrían tener una asociación potencial con la calidad de atención en el establecimiento como por ejemplo el total de mujeres en edad fértil, niveles de pobreza e inversiones en salud a nivel distrital y departamental.20

Para medir calidad de atención se utilizaron dos escalas, una de 101 puntos para el caso de los Hospitales y Centros de Capacitación, y otra de 71 puntos para establecimientos periféricos. La escala se construyó usando la metodología juicio de expertos; así, cada pregunta de exploración (item) del nivel de calidad fue puntuada según dos criterios: complejidad del proceso (considerado como el esfuerzo organizacional, operacional y de recursos que el establecimiento desarrolla para lograr su cumplimiento), y el beneficio potencial a la usuaria. Cada item tenía un puntaje de 0 a 3 por cada criterio; los puntajes otorgados por los expertos a cada Item según criterio eran sumados siendo el promedio de ambos el puntaje final considerado para cada uno de los items. La puntuación por cada componente y para la variable calidad de atención se obtuvo, para cada establecimiento, a través de sumatoria simple de los items incluidos en las categorias: uso de datos, manejo estandarizado, gestion y disponibilidad de recursos, educacion permanente en salud y relación proveedor usuario comunidad (Anexo 1). 


\section{Análisis de datos}

Se determinó el puntaje total de calidad de atención para cada uno de los 74 establecimientos estudiados y luego se procedió a agregarlos y compararlos según Grupos de estudio (P2000 y No P2000). Para ello se utilizaron estadísticos descriptivos de tendencia central, de dispersión y comparación de proporciones. La prueba U-Mann Whitney (la distribución del puntaje de calidad no seguía una distribución normal) fue utilizada para evaluar diferencias estadísticamente significativas entre grupos. Los datos de los conocimientos y actitudes de usuarias fueron analizados a través de tablas de contingencia según grupos de estudio (P2000 y No P2000), estimándose estadísticos como chi cuadrado, OR Maentel - Hanztel ajustado, intervalos de confianza al $95 \%$ y su nivel de significancia.22

Para determinar la contribución del Proyecto 2000 en el nivel de calidad, se construyó un modelo predictivo multivariado en el cual la variable dependiente fue el nivel de calidad de atención (medida en escala continua), las variables independientes (predictoras) consideradas fueron: el número de médicos, el número de enfermeras y obstetrices, la población de mujeres en edad fértil (MEF) del distrito, el nivel socioeconómico distrital (medido a través de la escala de Necesidades Básicas Insatisfechas), la participación del Proyecto 2000 y el nivel de complejidad del establecimiento. La técnica de análisis utilizada fue regresión lineal múltiple, se realizaron además pruebas de colinearidad, estimación y análisis de residuales, e interacción. En todo el análisis se consideró como nivel significativo un valor p menor de 0,05 .

Para el ingreso de datos en computadora se utilizó Epi-info versión 6.04, el análisis estadístico se realizó utilizando el software SPSS versión 10.0.

\section{Resultados}

\section{Calidad de atención}

El nivel de calidad de atención expresado en términos de la escala de 101 puntos para los Hospitales y Centros de Salud, muestra que la calidad es mayor en el Grupo P2000. El promedio de los puntajes de ese grupo fue 61,8 puntos (DS $=13,5$ puntos) en tan- to que el grupo de comparación (No P2000) fue 37,5 (DS $=9,9$ puntos), estas diferencias tienen un valor $\mathrm{p}<0,001$; esto representa que el nivel de calidad de atención es mayor en un 39\% en el grupo P2000 a nivel de Hospitales y Centros de Salud (Figura 2).

Figura 2

Diagrama de caja de los puntajes de calidad en Hospitales y Centros de Salud según grupo de estudio.

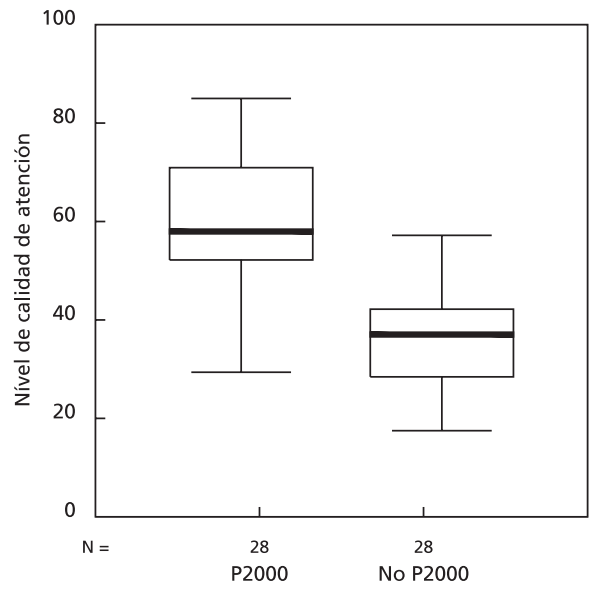

La diferencia de los niveles de calidad según componentes para cada grupo de estudio se muestran en la Tabla 1, se evidenció que en la totalidad de componentes el Grupo P2000 tuvo mayor nivel de desarrollo comparado con el Grupo No P2000 (diferencias estadísticamente significativas), siendo los componentes Educación permanente en salud (58\%), Manejo estandarizado (53\%) y Uso de datos (52\%) los que mostraron mayor diferencia entre los grupos. Comparando los puntajes de calidad alcanzados por el grupo de establecimientos del P2000 y el máximo puntaje esperado en cada componente, se observa que el mayor nivel alcanzado se observa en el componente Relación proveedor-usuario-comunidad con 15,81 puntos de un máximo posible de 19,2 (equivalente al $82 \%$ ). 
Puntajes de calidad de atención según componentes hospitales y centros de salud $(n=56)$.

\begin{tabular}{|c|c|c|c|c|c|}
\hline \multirow[t]{2}{*}{ Componente de Calidad } & \multirow{2}{*}{$\begin{array}{l}\text { Puntaje máximo } \\
\text { alcanzable }\end{array}$} & \multirow{2}{*}{$\begin{array}{c}\text { Grupo } \\
\text { Proyecto } 2000 \\
\bar{X} \pm d p\end{array}$} & \multirow{2}{*}{$\begin{array}{c}\begin{array}{c}\text { Grupo } \\
\text { No P2000 }\end{array} \\
\bar{X} \pm d p\end{array}$} & \multirow{2}{*}{$\begin{array}{c}\begin{array}{c}\text { Diferencia \% } \\
\text { entre grupos }\end{array} \\
\text { P2000 - NoP2000 }\end{array}$} & \multirow[t]{2}{*}{ Valor $\mathrm{p}^{*}$} \\
\hline & & & & & \\
\hline Educación permanente en salud & 10,80 & $3,85 \pm 2,41$ & $1,61 \pm 1,57$ & 58,0 & $<0,001$ \\
\hline Manejo estandarizado & 14,10 & $5,45 \pm 2,08$ & $2,57 \pm 1,52$ & 53,0 & $<0,001$ \\
\hline Uso de datos & 24,30 & $13,08 \pm 5,20$ & $6,23 \pm 3,36$ & 52,0 & $<0,001$ \\
\hline $\begin{array}{l}\text { Relación proveedor- usuario- } \\
\text { comunidad }\end{array}$ & 19,20 & $15,81 \pm 3,92$ & $9,51 \pm 2,29$ & 39,8 & $<0,001$ \\
\hline $\begin{array}{l}\text { Gestión y disponibilidad de } \\
\text { recursos }\end{array}$ & 33,22 & $21,63 \pm 7,11$ & $15,69 \pm 7,51$ & 27,0 & 0,004 \\
\hline Calidad de atención & 101,62 & $61,80 \pm 13,50$ & $37,50 \pm 9,90$ & 39,0 & $<0,001$ \\
\hline
\end{tabular}

* Calculado con test U- Mann Whitney.

Al comparar los puntajes alcanzados por cada uno de los items según grupos de estudio se encontró que, en general, el grupo P2000 tiene mayor puntaje (Tabla 2), siendo estas diferencias en la mayoría de casos estadísticamente significativas. Así, en el componente Uso de datos se aprecia que de los 13 items de estudio, el P2000 tiene un mayor puntaje significativo en 8 items (62\%) como por ejemplo el uso adecuado de la historia clínica materno perinatal, reuniones de discusión de resultados, publicación y difusión de informes, y toma de decisiones que benefician a las usuarias de servicios. En el componente Manejo estandarizado, en el $50 \%$ de los items estudiados los puntajes de calidad son mayores en el grupo P2000; sin embargo, en ambos grupos es notable la baja performance en el uso adecuado de los protocolos neonatales/pediátricos. El 57\% de los items del componente Gestión y disponibilidad de recursos en el grupo P2000 tienen mayor puntaje que el grupo de comparación, siendo los aspectos de planeamiento de actividades basados en calidad de atención y la existencia de insumos para la atención de complicaciones obstétricas y neonatales los de mayor diferencia. En el 60\% de items del componente Relación proveedor-usuario-comunidad, el puntaje alcanzado por el grupo P2000 también es mayor, siendo las actividades relacionadas con los agentes comunitarios de salud y los procesos de atención a las usuarias las que tuvieron mayor rendimiento. 
Tabla 2

Calidad de Atención en Hospitales y Centros de Salud P2000/ No P2000 ( $n=56$ )

\begin{tabular}{|c|c|c|c|}
\hline \multirow[t]{2}{*}{ Componente } & $\begin{array}{l}\text { Grupo Proyecto } \\
2000\end{array}$ & Grupo No P2000 & \multirow[t]{2}{*}{ Valor $\mathbf{p}(*)$} \\
\hline & $\bar{X} \pm d p$ & $\bar{X} \pm d p$ & \\
\hline \multicolumn{4}{|l|}{ Uso de datos: Item (estándar) } \\
\hline Seguimiento domiciliario de gestantes ( 2,8 puntos) & $1,55 \pm 0,26$ & $0,67 \pm 0,25$ & 0,022 \\
\hline Investigaciones operativas realizadas en el establecimiento (2) & $0,42 \pm 0,13$ & $0,18 \pm 0,09$ & 0,231 \\
\hline $\begin{array}{l}\text { Seguimiento de los acuerdos de las reuniones de los equipos de gestión } \\
\text { del establecimiento }(1,6)\end{array}$ & $0,73 \pm 0,17$ & $0,19 \pm 0,13$ & 0,023 \\
\hline Llenado correcto de la historia clínica $(1,8)$ & $0,87 \pm 0,08$ & $0,11 \pm 0,11$ & $<0,001$ \\
\hline Diagnóstico correcto de anemia gestacional $(2,3)$ & $1,22 \pm 0,18$ & $0,86 \pm 1,7$ & 0,193 \\
\hline $\begin{array}{l}\text { Difusión de estadísticas de partos institucionales al personal del } \\
\text { establecimiento }(1,6)\end{array}$ & $1,2 \pm 0,14$ & $0,75 \pm 0,2$ & 0,071 \\
\hline $\begin{array}{l}\text { Reuniones de análisis de información de los equipos implementadores de } \\
\text { la calidad }(1,8)\end{array}$ & $0,93 \pm 0,13$ & $0,66 \pm 0,15$ & 0,206 \\
\hline $\begin{array}{l}\text { Difusión de las estadísticas de muertes perinatales al personal del } \\
\text { establecimiento }(1,6)\end{array}$ & $1,07 \pm 0,16$ & $0,19 \pm 0,13$ & $<0,001$ \\
\hline $\begin{array}{l}\text { Reuniones de problematización con acuerdos de beneficio directo al } \\
\text { usuario }(1,7)\end{array}$ & $0,48 \pm 0,06$ & $0,34 \pm 0,10$ & 0,065 \\
\hline Reuniones de problematización con acuerdos de tipo organizacional $(1,7)$ & $0,85 \pm 0,08$ & $0,83 \pm 0,15$ & 0,709 \\
\hline $\begin{array}{l}\text { Registro de gestantes que no acuden a la cita de Control Prenatal } \\
\text { institucional }(2,2)\end{array}$ & $1,93 \pm 0,15$ & $1,55 \pm 0,25$ & 0,184 \\
\hline Uso de la historia clínica materno perinatal $(1,5)$ & $1,5 \pm 0,0$ & $0,09 \pm 0,00$ & $<0,001$ \\
\hline $\begin{array}{l}\text { Difusión de las estadísticas de muertes maternas al personal del } \\
\text { establecimiento }(1,7)\end{array}$ & $1,13 \pm 0,17$ & $0,20 \pm 0,14$ & $<0,001$ \\
\hline \multicolumn{4}{|l|}{ Manejo estandarizado: item (estándar) } \\
\hline $\begin{array}{l}\text { Disponibilidad de tecnologías perinatales en los consultorios de control } \\
\text { obstétrico }(2,1)\end{array}$ & $1,75 \pm 0,12$ & $1,06 \pm 0,16$ & $<0,001$ \\
\hline Existencia de protocolos de afecciones obstétricas de importancia $(2,4)$ & $0,98 \pm 0,11$ & $0,29 \pm 0,16$ & $<0,001$ \\
\hline $\begin{array}{l}\text { Existencia de protocolos de afecciones neonatales / pediátricos de } \\
\text { importancia }(2,4)\end{array}$ & $1,35 \pm 0,09$ & $0,44 \pm 0,13$ & $<0,001$ \\
\hline $\begin{array}{l}\text { Disponibilidad de petitorios de medicamentos en los consultorios } \\
\text { externos }(1,8)\end{array}$ & $0,22 \pm 0,08$ & $0,19 \pm 0,08$ & 0,860 \\
\hline Uso correcto de protocolos obstétricos $(2,7)$ & $0,98 \pm 0,12$ & $0,76 \pm 0,13$ & 0,180 \\
\hline Uso correcto de protocolos neonatales / pediátricos $(2,7)$ & $0,25 \pm 0,09$ & $0,37 \pm 0,16$ & 0,719 \\
\hline \multicolumn{4}{|l|}{ Gestion y disponibilidad de recursos: item (estándar) } \\
\hline $\begin{array}{l}\text { Disponibilidad de medicamentos genéricos trazadores en la farmacia del } \\
\text { establecimiento }(2,4)\end{array}$ & $2,03 \pm 0,06$ & $1,77 \pm 0,09$ & 0,024 \\
\hline Disponibilidad de médicos en el establecimiento $(2,9)$ & $1,58 \pm 0,23$ & $1,51 \pm 0,26$ & 0,786 \\
\hline $\begin{array}{l}\text { Plan operativo local incluye aspectos relacionados con la calidad de } \\
\text { atención }(1,6)\end{array}$ & $1,05 \pm 0,08$ & $0,55 \pm 0,09$ & $<0,001$ \\
\hline $\begin{array}{l}\text { Disponibilidad de consultorios de atención prenatal e infantil en el } \\
\text { establecimiento }(2,2)\end{array}$ & $1,56 \pm 0,21$ & $0,80 \pm 0,20$ & 0,015 \\
\hline
\end{tabular}


continuação

\begin{tabular}{|c|c|c|c|}
\hline \multirow[t]{2}{*}{ Componente } & \multirow{2}{*}{$\begin{array}{l}\text { Grupo Proyecto } \\
2000 \\
\bar{X} \pm d p\end{array}$} & Grupo No P2000 & \multirow[t]{2}{*}{ Valor $p(*)$} \\
\hline & & $\bar{X} \pm d p$ & \\
\hline $\begin{array}{l}\text { Existencia de objetivos comunes en el equipo de gestión del } \\
\text { establecimiento }(1,8)\end{array}$ & $0,8 \pm 0,11$ & $0,39 \pm 0,07$ & 0,006 \\
\hline Existencia de insumos de transfusión sanguínea $(2,8)$ & $2,45 \pm 0,15$ & $2,11 \pm 0,22$ & 0,151 \\
\hline Disponibilidad de personal de laboratorio $(2,2)$ & $1,32 \pm 0,15$ & $1,26 \pm 0,18$ & 0,793 \\
\hline Existencia de insumos para emergencias neonatales $(2,6)$ & $2,22 \pm 0,06$ & $1,83 \pm 0,15$ & 0,034 \\
\hline $\begin{array}{l}\text { Disponibilidad de insumos para el manejo de las neumonía en el menor de } \\
1 \text { año }(2,4)\end{array}$ & $1,61 \pm 0,11$ & $1,45 \pm 0,16$ & 0,378 \\
\hline Funcionalidad de sala de operaciones $(2,7)$ & $2,03 \pm 0,24$ & $2,06 \pm 0,29$ & 0,915 \\
\hline Disponibilidad de personal entrenado en brindar anestesia (3) & $1,75 \pm 0,30$ & $1,53 \pm 0,32$ & 0,026 \\
\hline $\begin{array}{l}\text { Capacidad del laboratorio local para realizar descarte de sífilis en } \\
\text { gestantes }(2,1)\end{array}$ & $2,01 \pm 0,09$ & $1,48 \pm 0,24$ & 0,794 \\
\hline Disponibilidad de enfermeras / obstetrices en el establecimiento (2) & $1,23 \pm 0,13$ & $1,21 \pm 0,14$ & 0,045 \\
\hline Existencia de insumos para emergencias obstétricas $(2,52)$ & $1,97 \pm 0,12$ & $1,69 \pm 0,13$ & 0,009 \\
\hline \multicolumn{4}{|l|}{ Educacion permanente en salud: item (estándar) } \\
\hline $\begin{array}{l}\text { Nivel de discusión de las reuniones de los Comités de Prevención de } \\
\text { Mortalidad Materna Perinatal }(1,9)\end{array}$ & $0,48 \pm 0,08$ & $0,17 \pm 0,06$ & 0,009 \\
\hline Existencia del Comité de Prevención de Mortalidad Materna Perinatal $(1,5)$ & $1,17 \pm 0,11$ & $0,91 \pm 0,11$ & 0,05 \\
\hline Plan de entrenamiento de personal nuevo & $0,60 \pm 0,18$ & $0,12 \pm 0,12$ & 0,046 \\
\hline Capacitación del personal nuevo $(2,3)$ & $0,63 \pm 0,20$ & $0,14 \pm 0,14$ & 0,046 \\
\hline $\begin{array}{l}\text { Reuniones de problematización de los comités de prevención de } \\
\text { mortalidad materna }(1,6)\end{array}$ & $0,63 \pm 0,11$ & $0,36 \pm 0,12$ & 0,074 \\
\hline Momento de la última reunión de los CPMM $(1,5)$ & $0,58 \pm 0,13$ & $0,25 \pm 0,11$ & 0,032 \\
\hline \multicolumn{4}{|l|}{ Relación proveedor usuario comunidad: item (estándar) } \\
\hline Capacitación de los agentes comunitarios de salud $(2,2)$ & $1,58 \pm 0,19$ & $0,28 \pm 0,14$ & $<0,001$ \\
\hline Uso de la información de los buzones de sugerencias $(2,1)$ & $1,11 \pm 0,13$ & $0,54 \pm 0,09$ & 0,003 \\
\hline Limpieza de los servicios higiénicos $(1,7)$ & $1,22 \pm 0,11$ & $1,03 \pm 0,16$ & 0,336 \\
\hline Existencia de buzón de sugerencias en el establecimiento $(1,4)$ & $1,34 \pm 0,06$ & $1,07 \pm 0,15$ & 0,065 \\
\hline $\begin{array}{l}\text { Implementación de mejoras en el establecimiento tomando como base } \\
\text { sugerencias del buzón }(2,3)\end{array}$ & $1,44 \pm 0,23$ & $0,41 \pm 0,22$ & 0,005 \\
\hline $\begin{array}{l}\text { Referencias de pacientes al establecimiento realizadas por los agentes } \\
\text { comunitarios de salud }(2,8)\end{array}$ & $2,16 \pm 0,20$ & $0,86 \pm 0,19$ & $<0,001$ \\
\hline Seguimiento de pacientes con neumonía $(2,7)$ & $2,28 \pm 0,25$ & $1,89 \pm 0,27$ & 0,675 \\
\hline Funcionalidad del Radar de gestantes $(2,3)$ & $1,44 \pm 0,23$ & $0,68 \pm 0,26$ & 0,039 \\
\hline Limpieza de la sala de espera $(1,7)$ & $1,70 \pm 0,00$ & $1,60 \pm 0,10$ & 0,235 \\
\hline Señalización en el interior del establecimiento $(1,6)$ & $1,43 \pm 0,06$ & $1,43 \pm 0,07$ & 1,00 \\
\hline Calidad de atencion & $61,8 \pm 13,5$ & $37,5 \pm 9,90$ & $<0,001$ \\
\hline
\end{tabular}

*Calculado con test U Mann-Whitney. 
En los establecimientos de salud periféricos, se encontró que el nivel de calidad fue mayor en el Grupo P2000 (Media: 30,61 puntos, DS 7,41) en relación al Grupo No P2000 (Media: 24,95 puntos, DS 7,43), las diferencias entre ambos son del $18 \%$. (Figura 3) pero sin mostrar diferencias estadísticamente significativos $(p=0,125)$. Adicionalmente, los puntajes de calidad según componentes y grupos de estudio mostraron diferencias discretas (Tabla 3).

\section{Figura 3}

Diagrama de caja de los puntajes de calidad en establecimientos periféricos según grupo de estudio, 2000.

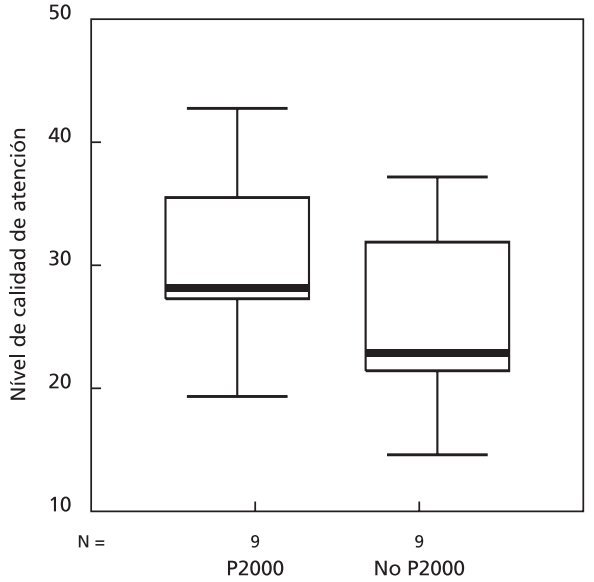

Tabla 3

Puntajes de calidad de atención según componentes y grupos de estudio establecimientos periféricos

\begin{tabular}{|c|c|c|c|c|}
\hline \multirow{2}{*}{ Componente de calidad } & \multirow[t]{2}{*}{$\begin{array}{l}\text { Puntaje máximo } \\
\text { alcanzable }\end{array}$} & $\begin{array}{l}\text { Grupo Proyecto } \\
2000\end{array}$ & $\begin{array}{l}\text { Grupo No } \\
\text { P2000 }\end{array}$ & \multirow{2}{*}{ Valor $p^{*}$} \\
\hline & & $\bar{X} \pm d p$ & $\bar{X} \pm d p$ & \\
\hline Uso de datos & 20 & $5,83 \pm 3,16$ & $4,69 \pm 3,41$ & 0,470 \\
\hline Manejo estandarizado & 3,9 & $2,19 \pm 1,19$ & $2,08 \pm 0,54$ & 0,802 \\
\hline $\begin{array}{l}\text { Relación proveedor- usuario- } \\
\text { comunidad }\end{array}$ & 20,8 & $13,84 \pm 4,32$ & $11,04 \pm 2,86$ & 0,125 \\
\hline Educación permanente en salud & 8,8 & $1,05 \pm 1,04$ & $0,83 \pm 1,02$ & 0,663 \\
\hline $\begin{array}{l}\text { Gestión y disponibilidad de } \\
\text { recursos }\end{array}$ & 17,82 & $7,69 \pm 1,72$ & $6,31 \pm 2,07$ & 0,143 \\
\hline Calidad de atención & 71,32 & $30,61 \pm 7,41$ & $24,95 \pm 7,43$ & 0,125 \\
\hline
\end{tabular}

* Calculado con test U Mann-Whitney. 


\section{Conocimientos y actitudes de las usuarias}

Se midió el grado de conocimientos de signos de alarma obstétricos en las gestantes que egresaron de la consulta prenatal, los resultados en general muestran que la proporción de mujeres que conocían los signos de alarma es mayor en el Grupo P2000, estas diferencias son significativas en tres (fiebre, sangrado vaginal y pies/manos hinchadas) de los siete signos (Tabla 4). El número promedio de signos de alarma reportado por cada gestante del Grupo P2000 es de 3.58 mientras que en el Grupo No P2000 este promedio es $2,52(\mathrm{p}<0,05)$. Nótese el relativamente bajo porcentaje de mujeres que reportaron la ausencia de movimiento fetal como signo de alarma, sobretodo en el Grupo No P2000.

En el Grupo P2000, el 78\% de las encuestadas reconoce al sangrado vaginal como signo de alarma, mientras que en el grupo No P2000 dicha proporción llega al $61 \%$ o cual significa que la gestante que egresa de los consultorios de atención prenatal de los establecimientos P2000 tuvieron dos veces mas probabilidades $(\mathrm{OR}=2,32$, IC95\%: 1,21-5,61, $\mathrm{p}=$ $0,038)$ de reconocer al sangrado vaginal como signo de alarma obstétrico que las gestantes del Grupo No P2000. Situación similar ocurre con el reconocimiento de los signos manos /pies hinchados y fiebre.

En relación a las actitudes de las ex-usuarias de los servicios obstétricos (143 entrevistadas) se encontró que la intención de uso de los servicios de parto institucional muestra diferencias significativas entre grupos, así una ex-usuaria de los servicios maternos de los establecimientos del P2000 tuvo dos veces más posibilidades de acudir al establecimiento para tener parto institucional $(\mathrm{OR}=2,26, \mathrm{IC} 95 \%$ : $1,01-5,04, \mathrm{p}=0,046)$ en comparación al Grupo No P2000.

Nivel de conocimientos de signos de alarma en usuarias de servicios según grupos de comparación.

\begin{tabular}{lcccrrr}
\hline Grupo & $\begin{array}{c}\text { Manos/pies } \\
\text { hinchados }\end{array}$ & Cefalea & $\begin{array}{c}\text { Sangrado } \\
\text { vaginal }\end{array}$ & Fiebre & $\begin{array}{c}\text { Dolor } \\
\text { abdominal }\end{array}$ & $\begin{array}{c}\text { Perdida de } \\
\text { liquido } \\
\text { movimiento } \\
\text { fetal }\end{array}$ \\
\hline P2000 $(n=72)$ & 55,1 & 47,8 & 78,3 & 52,2 & 39,1 & 31,0 \\
No P2000 $(n=51)$ & 35,3 & 31,4 & 60,8 & 21,6 & 33,3 & 0,4 \\
Valor $p^{*}$ & 0,032 & 0,070 & 0,037 & 0,001 & 0,515 & 0,069 \\
\hline
\end{tabular}

* Calculado con test U Mann-Whitney.

\section{Contribución del Proyecto 2000 sobre la calidad de atención}

Los resultados de la intervención en los 56 Hospitales y Centros de Salud evaluados, mostraron que el modelo predictivo de regresión lineal múltiple explicó el 74,6\% de la varianza de la variable calidad de atención $\left(\mathrm{R}^{2}\right.$ ajustado $\left.=0,746\right)$. Las variables explicatorias de significancia fueron el Proyecto 2000 (coeficiente beta estandarizado $=0,652, \mathrm{p}<0,001)$ y la disponibilidad de obstetrices y enfermeras (coeficiente beta estandarizado $=0,368, \mathrm{p}<0,001)$. Esto significa que el implementar el modelo de calidad del Proyecto 2000 incrementaría el nivel de la calidad de atención en un 65\% $(0,652)$; en el caso de las obstetrices y enfermeras el incremento en un profesional ocasionaría un aumento de 0,368 unidades en el nivel de calidad. Esto significaría que la contribución del P2000 en incrementar el nivel de calidad de un establecimiento es aproximadamente el doble que la disponiblidad única de obstetrices y enfermeras. Otra variable predictora identificada fue el NBI, al incrementarla en una unidad (es decir, mayor nivel de pobreza) el nivel de calidad de atención disminuiría en $14 \%$; lo cual significaría que la calidad de atención es más factible de desarrollarla en lugares con menores limitaciones de recursos. Al aplicar las pruebas de interacción la potencia del modelo estadístico no se modificó (Tabla 4). 
Análisis multivariado de nivel de calidad de atención en hospitales y centros de salud cabeceras de red según variables explicatorias.*

\begin{tabular}{lcrr}
\hline Variable explicatoria & B (error estándar) & B estandarizado & Valor $\mathbf{p}$ \\
\hline Constant & $38,66(5,37)$ & 0,097 & 0,408 \\
Médicos & $0,151(0,181)$ & 0,368 & $<, 001$ \\
Obstetrices y enfermeras & $0,512(0,146)$ & $-0,103$ & 0,285 \\
Mujeres en edad fértil & $3,86 \mathrm{E}-05$ & 0,652 & 0,001 \\
Intervención Proyecto 2000 & $21,46(2,29)$ & $-0,189$ & 0,049 \\
Necesidades básicas insatisfechas (NBI) & $-0,139(0,069)$ & & \\
R2 (error estándar) & 0,683 & & \\
R2 ajustado (error estándar) & 0,746 & 56 & \\
Establecimientos $(n)$ & & & \\
\hline
\end{tabular}

* Regresión Lineal Múltipla; ANOVA F = 29,35 ( $p<0,001)$.

\section{Discussion}

\section{Calidad de atención en establecimientos}

La calidad de atención es una de las líneas de acción sanitarias más fuertemente impulsadas por las reformas de los sistemas de salud en América Latina y muy en especial en el área de salud reproductiva.9,10 Se postula que al mejorar la calidad de atención se promueve una mayor utilización de los servicios y un mejor estado de salud de los usuarios y la población en general.23 En Nepal, en un estudio poblacional reciente, se encontró que aquellos establecimientos con mayores niveles de calidad tuvieron mayor utilización de los servicios de consulta prenatal y mayores coberturas de vacunación infantil. ${ }^{23}$ Parvez, ${ }^{24}$ encontró una relación directa entre calidad y organización de los servicios de salud y su utilización por las mujeres, sobretodo en establecimientos periféricos; asimismo los programas de calidad que dan énfasis al trabajo extramural con seguimiento y captación de gestantes mejoran la cobertura de CPN y parto institucional. ${ }^{25}$ También se ha encontrado que el CPN, 7,26-28 y sobretodo la atención del parto institucional, tienen influencia en la reducción de la morbimortalidad materna.29,30 Problemas derivados del sistema de salud como la oportunidad del tratamiento y la calidad del mismo influyen decisivamente en la ocurrencia de muertes maternas. 1

El actual estudio contribuye a generar información acerca de los efectos de una intervención en salud sobre la calidad de atención en los servicios obstétricos, sobretodo en el momento actual en que la agenda de la salud internacional impulsa el desarrollo y sostenibilidad de estrategias de intervención efectivas y eficaces en salud reproductiva para un problema tan sentido en los países en vías en desarrollo como la mortalidad materna. 5,11

Los resultados de este estudio muestran que el nivel de calidad de atención es significativamente mayor en los establecimientos que recibieron directamente la intervención PMC. Apreciándose que el personal de salud de los Hospitales y Centros de Salud brindan mejor atención a los usuarios y cumplen con los estándares de los principales procesos de atención materno perinatal. Aun así existen un conjunto de procesos que pueden fortalcerse.

La mejora de la calidad de los servicios contribuiría en la protección de la salud de la población; Magadi et al. ${ }^{31}$ han reportado que el riesgo de muerte materna se ve afectado por los niveles de calidad en los servicios de salud, así la tasa de mortalidad hospitalaria en Kenya con alta calidad es de cuatro muertes por 1000 mientras que en hospitales con deficiente nivel de calidad este indicador se eleva a 280 muertes por 1000 (tasas ajustadas por edad, nivel socioeconómico y acceso a control prenatal); Acharya et al. ${ }^{23}$ sugieren que el mejoramiento de los servicios de salud a nivel hospitalario contribuye a asegurar las vidas de las mujeres que viven próximas a ella, es decir la calidad de los servicios se constituye en un factor protector de la salud de la población que accede a ella. Esto apunta a sustentar que la calidad en los establecimientos con PMC está contribuyendo a garantizar la calidad de vida de su población. 
Uno de los aspectos más fuertemente impulsados por el Proyecto, fue el análisis crítico y regular de los procesos de atención a través de las sesiones de problematización a cargo de los equipos multidisciplinarios mediante el uso de técnicas participativas como la tormenta de ideas, priorización de problemas, análisis de causa efecto, implementación de soluciones viables, entre otros. Este esfuerzo se enmarca dentro del componente EPS, el cual se observa que tuvo un nivel mayor en 58\% a favor de los establecimientos del Proyecto. Similar situación se observa en el componente UD, en donde la utilización de la información para la toma de decisiones es trascendental en la rutina diaria de la gestión de servicios, sobre todo en los establecimientos del P2000 (puntaje equivalente al 53\% del estándar).

El modelo implementado por el Proyecto tuvo un fuerte desarrollo de estrategias comunicacionales entre el proveedor y las usuarias, promovió un intenso trabajo extramural, visitas de captación y seguimiento de gestantes, trabajo con los agentes comunitarios de salud y mejora de las referencias por niveles de atención lo que favoreció una mayor aceptabilidad y continuidad de los servicios. Por ello es que el componente RPUC, es el que muestra mayor nivel de desarrollo ( $82 \%$ en relación al su estándar) en el Grupo P2000. Estos aspectos son de importancia ya que influyen en la salud materna, por ejemplo, en un estudio desarrollado en Bangladesh se evidenció la contribución de los servicios de atención periféricos con obstetrices capacitadas en brindar funciones obstétricas básicas (atención de parto y manejo inicial de complicaciones y referencia precoz) y atención extramural, sobre la reducción de la mortalidad materna. 12 Asimismo se ha evidenciado la relevancia del primer nivel de atención en generar mejores condiciones de accesibilidad para las gestantes, mayores niveles de referencia oportuna ante complicaciones obstétricas ${ }^{32}$ y disminución de la mortalidad materna. ${ }^{33,34}$ Esta información le añade valor social al esfuerzo por alcanzar niveles de calidad de atención óptimos en lo servicios de salud.

Adicionalmente, en el Grupo P2000 el componente RPUC impulsó la adaptación de los servicios de atención de parto, considerando las características socioculturales de las gestantes y del entorno familiar y comunitario, sobre todo en zonas rurales de sierra y selva; estas acciones podrían explicar los resultados encontrados en los niveles de calidad de este componente. Estas acciones tendrían traducción en una mayor aceptación y utilización de los servicios de parto, tal como lo señaló Bloom et al.,25 quienes en un estudio realizado en la zona urbanomarginal de la India encontraron que las barreras sociocultur- ales y antropológicas deben ser consideradas en la definición de los servicios obstétricos, especialmente del parto, a fin de favorecer su aceptación y uso.

\section{Conocimientos y actitudes de las usuarias}

Uno de los determinantes de la utilización de los servicios de salud obstétricos es el grado de conocimientos de las mujeres en aspectos básicos de salud materna. Además existe correlación entre la actitud de las usuarias y la búsqueda de atención y uso de servicios. ${ }^{35}$ En el presente estudio, los signos de alarma obstétricos son reconocidos en mayor proporción por las gestantes que egresan de los consultorios de atención prenatal de los establecimientos del Proyecto 2000, sobretodo de signos como fiebre, manos/pies hinchados y sangrado vaginal, los cuales están relacionados con la presencia de infección/sepsis en la gestación, enfermedad hipertensiva del embarazo y hemorragia obstétrica respectivamente, entidades que constituyen las principales causas de mortalidad materna en el Perú.36,37 Estas diferencias podrían deberse, entre otros aspectos, a la estrategia de comunicación interpersonal y, a los procedimientos de orientación y consejería desarrollados en los establecimientos del Proyecto 2000. Dicha estrategia consistió en que el proveedor de servicios establecía eslabones comunicacionales con las usuarias durante la consulta prenatal y las visitas domiciliarias; proporcionando orientaciones a las gestantes sobre autocuidado de la salud, reconocimiento precoz de signos de alarma, consejos nutricionales, orientaciones psicoafectivas, cuidados del bebe, entre otros. Asimismo se impulsó un fuerte trabajo de acción extramural de prevención y promoción de la atención del CPN y el parto institucional. Es sabido que el grado de conocimientos en salud tiene cierta asociación con el nivel educativo y socioeconómico de la mujer, y estos a su vez influyen en el uso de los servicios obstétricos; 25 en este estudio no se ha realizado un ajuste de tales variables durante el análisis, sin embargo, las características del muestreo y el similar patrón educativo y socioeconómico de las usuarias en los establecimientos estudiados de ambos grupos podría minimizar tal influencia, si es que esta existiese.

Las actitudes tienen cierta influencia en la utilización de los servicios de salud. ${ }^{38}$ Gestantes con una intención favorable por el uso de servicios de salud, tienen más probabilidades de efectivizarlo que aquellas que no tienen tal intención. ${ }^{29}$ En el presente estudio, las diferencias encontradas a favor de las usuarias de establecimientos del Grupo P2000 en la intención de usar los servicios de parto institucional, 
hacen presumir que su verdadero uso sería mayor en tales establecimientos. Considerando que la atención del parto por profesional de salud entrenado es una de las variables más directamente relacionadas con la reducción de la mortalidad materna, ${ }^{30,39}$ el Proyecto 2000 estaría influyendo positivamente en tal sentido, si es que efectivamente está logrando el incremento del uso de los servicios de parto institucional. Por tanto, la relación entre intención de uso y prácticas es un aspecto de trascendencia que ameritaría ser estudiado en mayor profundidad.

Por otro lado, la implementación de PMC requiere de un trabajo compartido, estrecho y regular entre los niveles de gestión, los proveedores y usuarios de la atención, su no articulación puede afectar los resultados en los niveles de calidad tal como ocurrió en los establecimientos perfiféricos del presente estudio.

\section{Contribución del Proyecto 2000 sobre la calidad} de atención

El análisis multivariado realizado proporciona resultados, con un $75 \%$ de certeza, que señalan que de los predictores estudiados, la intervención Proyecto 2000 está más asociada a la calidad de atención, seguida de la disponibilidad de recursos humanos. Esto robustece el concepto de que para obtener calidad la disponibilidad de recursos humanos es importante, pero más lo es el desarrollar procesos de mejora continua interfuncionales, integrales e interrrelacionados que promuevan el trabajo el equipo y el enfoque hacia las usuarias. Por otro lado, según nuestros hallazgos la limitación de recursos (nivel de pobreza) es un factor a considerar dentro de las políticas de implementación de programas de mejora de calidad.

Se estima que los mayores niveles de calidad favorezcan a las usuarias y contribuyan en la mejora de su estado de salud a través de una reducción en el riesgo de fallecer durante el embarazo, parto y puerperio. Estudios sobre la calidad de atención en otras latitudes del mundo 29,32,40 con altas tasas de mortalidad materna, han encontrado asociación positiva entre el nivel de calidad y el beneficio de la población usuaria de hospitales y centros de salud periféricos. $25,28,29,32,34$

La evidencia, de otros $11,23,41$ y del presente estudio, orienta a los decisores de política a direccionar la inversión sanitaria hacia la mejora de la calidad de los servicios. La oferta de servicios obstétricos de calidad y el incremento de la accesibilidad oportuna de las gestantes a tales servicios, son las estrategias sanitarias más efectivas que contribuyen en la dis- minución de los niveles del complejo problema sanitario denominado mortalidad materna. $11,32,42,43$

\section{Limitaciones del estudio}

La metodología empleada ha tratado, en lo posible, de controlar los efectos de posibles sesgos y variables confusoras que interactúan sobre el nivel de calidad de los establecimientos; para tal fin se estratificaron los departamentos de comparación y se realizó un muestreo aleatorio. Posteriormente en la fase de análisis se aplicaron técnicas de análisis multivariado con la intención de controlar posibles confusores. Sin embargo, no se han medido variables del contexto ni de otras intervenciones en salud que se han desarrollado simultáneamente en los establecimientos estudiados sobre todo en los recursos humanos, infraestructura física, el equipamiento de los servicios de radiodiagnóstico y laboratorio, hospitalización y transporte de pacientes. Sin embargo, estos grandes proyectos tuvieron aplicación nacional, por lo que consideramos que sus efectos se distribuyen en ambos grupos de estudio. Adicionalmente, otras intervenciones han desarrollado acciones en gerencia de redes de establecimientos y en salud materna; sin embargo estas se desarrollaron sobretodo en los establecimientos del Grupo No P2000. Por ello, consideramos que los resultados encontrados en el presente estudio no se invalidan por la simultaneidad de otras intervenciones. El tamaño muestral fue una limitante para estudiar mayor número de variables predictoras por lo que nos limitamos a incluir en el modelo sólo aquellas con mayor correlación con el nivel de calidad. Dichas consideraciones y el muestreo empleado contribuyen a robustecer la validez interna de los resultados. Situación similar ocurre con el nivel inferencial de los resultados. Sin embargo, el número de usuarias entrevistadas en el estudio del conocimientos de signos de alarma e Intención de uso (ver intervalos de confianza al 95\%) podríanse optimizar en un estudio posterior, a fin de caracterizar con mayor precisión la magnitud de las diferencias encontradas.

\section{Conclusiones}

A la luz de los hallazgos, existe un mayor nivel de calidad en los Hospitales y Centros de salud donde intervino el Proyecto siendo las diferencias relevantes a nivel global y por componentes; asimismo las usuarias de los servicios de atención prenatal tienen mayor probabilidades de reconocer los signos de alarma obstétricos y reportan mayor aceptación e 
intención de uso de los servicios de parto institucional. Todo esto contribuiría a su mejor estado de salud. El nivel de calidad de atención de los establecimientos se ve influenciado por: el PMC implementado por el Proyecto y por la disponibilidad de profesionales de salud. Pareciera que en lugares donde existen menores recursos disponibles se requiere de mayores esfuerzos para el desarrollo de la calidad de los servicios.

\section{Agradecimientos}

Los autores desean agradecer el aporte financiero del gobierno del Perú y de USAID por la implementación del Proyecto 2000 y la realización del estudio de evaluación, también desean reconocer los valiosos aportes del Lic. Francisco de la Cruz en el proceso de construcción de la base y análisis estadístico, agradecimientos finales a la Lic. Gaby Caro (OPS-Perú) por su aporte en la revisión de las referencias bibliográficas y a la Dra. Luciana Chagas (OPS Brasil) por la revisión global del manuscrito, y a todo el personal de salud de los hospitales estudiados, quienes con su esmerada y continua labor hicieron del Proyecto 2000 una realidad. 
Tabla de puntuacion de las variables de calidad: sesion de expertos.

Componente

\section{Puntuacion por expertos}

Complejidad Influencia sobre el usuario

Uso de datos $(n=13)$ : items

Gestantes que no acuden al CPN y que son seguidas en domicilio

Investigaciones operativas en el establecimiento

Seguimiento de los acuerdos de las reuniones

Llenado adecuado de la HCMP

Diagnóstico correcto de anemia gestacional

Difusión de estadísticas partos institucionales

Reuniones del personal del establecimiento para análisis de

información

Difusión de estadísticas muertes perinatales

Acuerdos en las reuniones de análisis

Acuerdos en beneficio directo del usuario

Reporte de gestantes que no acuden a CPN

Uso de historia clínica materno perinatal

Difusión de estadísticas muertes maternas

Manejo estandarizado $(n=6)$ : Items

Disponibilidad de tecnologías perinatales en los consultorios obstétricos

Disponibilidad y reconocimiento de protocolos de problemas

clínicos obstétricos

Uso de protocolos obstétricos

Disponibilidad y reconocimiento de protocolos de problemas

clínicos neonatal e infantil

Disponibilidad de petitorio de medicamentos esenciales en los consultorios

Uso de protocolos neonatales/pediátricos

Gestion y disponibilidad de recursos $(n=14)$ : Items

Disponibilidad de medicamentos genéricos trazadores

Disponibilidad de médicos

Calidad de contenido del plan operativo local

Disponibilidad de consultorios materno infantiles en el establecimiento

Existencia de objetivos comunes en el equipo de gestión del establecimiento

Disponibilidad de insumos para transfusión sanguínea

Disponibilidad de personal en laboratorio

Disponibilidad de insumos para el manejo de las emergencias neonatales

Disponibilidad de insumos para el manejo de las neumonías en el menor de un año

\begin{tabular}{llllllllllll} 
A & B & C & D & E & A & B & C & D & E & Suma & Média \\
\hline 3 & 3 & 2 & 3 & 2 & 3 & 3 & 3 & 3 & 3 & 28 & 2,8 \\
& & & & & & & & & & & \\
1 & 3 & 2 & 2 & 3 & 2 & 1 & 2 & 2 & 2 & 20 & 3,0 \\
2 & 2 & 1 & 1 & 3 & 1 & 1 & 2 & 1 & 2 & 16 & 1,6 \\
1 & 1 & 1 & 2 & 2 & 2 & 3 & 2 & 2 & 2 & 18 & 1,8 \\
2 & 1 & 1 & 2 & 2 & 3 & 3 & 3 & 3 & 3 & 23 & 2,3 \\
1 & 3 & 1 & 2 & 2 & 2 & 1 & 2 & 1 & 1 & 16 & 1,6 \\
2 & 2 & 2 & 2 & 2 & 2 & 1 & 1 & 2 & 2 & 18 & 1,8 \\
& & & & & & & & & & & \\
1 & 3 & 2 & 2 & 2 & 2 & 1 & 1 & 1 & 1 & 16 & 1,6 \\
1 & 2 & 2 & 2 & 2 & 2 & 1 & 2 & 2 & 1 & 17 & 1,7 \\
& & & & & & & & & & 17 & 1,7 \\
2 & 2 & 1 & 3 & 2 & 2 & 2 & 3 & 3 & 2 & 22 & 2,2 \\
1 & 1 & 1 & 1 & 1 & 2 & 2 & 2 & 3 & 1 & 15 & 1,5 \\
2 & 3 & 2 & 2 & 2 & 2 & 1 & 1 & 1 & 1 & 17 & 1,7
\end{tabular}

Subtotal 24,3

$\begin{array}{llllllllllll}2 & 2 & 1 & 1 & 2 & 3 & 3 & 3 & 2 & 2 & 21 & 2,1 \\ 2 & 2 & 3 & 3 & 2 & 2 & 3 & 3 & 2 & 2 & 24 & 2,4 \\ 2 & 3 & 2 & 2 & 3 & 3 & 3 & 3 & 3 & 3 & 27 & 2,7 \\ 2 & 2 & 3 & 3 & 2 & 2 & 3 & 3 & 2 & 2 & 24 & 2,4 \\ 1 & 3 & 1 & 2 & 2 & 2 & 2 & 2 & 1 & 2 & 18 & 1,8 \\ 2 & 2 & 3 & 2 & 3 & 3 & 3 & 3 & 3 & 3 & 27 & 2,7 \\ & & & & & & & & & & & \\ \text { Subtotal } & 14,1\end{array}$

$\begin{array}{llllllllllll}2 & 3 & 2 & 3 & 2 & 3 & 2 & 2 & 3 & 2 & 24 & 2,4\end{array}$

$\begin{array}{llllllllllll}3 & 3 & 3 & 3 & 2 & 3 & 3 & 3 & 3 & 3 & 29 & 29\end{array}$

$\begin{array}{llllllllllll}2 & 3 & 2 & 2 & 1 & 1 & 1 & 2 & 1 & 1 & 16 & 1,6\end{array}$

$\begin{array}{llllllllllll}2 & 2 & 1 & 2 & 2 & 2 & 3 & 2 & 3 & 3 & 22 & 2,2\end{array}$

$\begin{array}{lllllllllllll}2 & 2 & 2 & 2 & 3 & 1 & 1 & 2 & 1 & 2 & 18 & 1,8\end{array}$

$\begin{array}{llllllllllll}3 & 3 & 3 & 3 & 2 & 2 & 3 & 3 & 3 & 3 & 28 & 2,8\end{array}$

$\begin{array}{llllllllllll}2 & 2 & 2 & 2 & 2 & 2 & 2 & 2 & 3 & 3 & 22 & 2,2\end{array}$

$\begin{array}{llllllllllll}2 & 2 & 2 & 2 & 2 & 2 & 2 & 2 & 3 & 3 & 22 & 2,2 \\ 2 & 2 & 2 & 3 & 2 & 3 & 3 & 3 & 3 & 3 & 26 & 2,6\end{array}$

$\begin{array}{llllllllllll}1 & 2 & 1 & 3 & 2 & 3 & 3 & 3 & 3 & 3 & 24 & 2,4\end{array}$

continua 
continuação

Puntuacion por expertos

Componente

Complejidad

Influencia sobre el usuario

Operatividad de la sala de operaciones

Disponibilidad de personal entrenado en brindar anestesia

Capacidad del laboratorio local para el descarte serológico

de sífilis

Disponibilidad de enfermeras / obstetrices

Disponibilidad de insumos para el tratamiento de

\begin{tabular}{cccccccccccc} 
A & B & C & D & E & A & B & C & D & E & Suma & Média \\
\hline 2 & 2 & 2 & 3 & 3 & 3 & 3 & 3 & 3 & 3 & 27 & 2,7 \\
3 & 3 & 3 & 3 & 3 & 3 & 3 & 3 & 3 & 3 & 30 & 3,0 \\
2 & 1 & 2 & 2 & 3 & 2 & 2 & 1 & 3 & 3 & 21 & 2,1 \\
& & & & & & & & & & & \\
2 & 1 & 2 & 1 & 1 & 3 & 2 & 2 & 3 & 3 & 20 & 2,0 \\
2 & 2 & 2 & 2 & 2 & 3 & 3 & 3 & 3 & 3 & 25 & 2,52 \\
& & & & & & & & & & & \\
Subtotal 33,22
\end{tabular}

\begin{tabular}{cccccccccccc} 
A & B & C & D & E & A & B & C & D & E & Suma & Média \\
\hline 2 & 2 & 2 & 3 & 3 & 3 & 3 & 3 & 3 & 3 & 27 & 2,7 \\
3 & 3 & 3 & 3 & 3 & 3 & 3 & 3 & 3 & 3 & 30 & 3,0 \\
2 & 1 & 2 & 2 & 3 & 2 & 2 & 1 & 3 & 3 & 21 & 2,1 \\
& & & & & & & & & & & \\
2 & 1 & 2 & 1 & 1 & 3 & 2 & 2 & 3 & 3 & 20 & 2,0 \\
2 & 2 & 2 & 2 & 2 & 3 & 3 & 3 & 3 & 3 & 25 & 2,52 \\
& & & & & & & & & & & \\
Subtotal 33,22
\end{tabular}

emergencias obstétricas

Educacion permanente en salud $(n=6)$ : Items

Aspectos organizacionales como tema central tratado en las $\quad \begin{array}{llllllllllll}2 & 2 & 2 & 2 & 3 & 1 & 1 & 2 & 2 & 2 & 19 & 1,9\end{array}$

reuniones de los Comités de Prevención

de Mortalidad Materna Perinatal (CPMMP)

Existencia y reconocimiento local del CPMMP

Plan de entrenamiento de personal nuevo al establecimiento

Capacitación del personal nuevo

Reuniones del CPMMP

Tiempo de la última reunión de los CPMMP

Relación proveedor usuario comunidad ( $n=9$ ): items

Capacitación de los agentes comunitarios de salud (ACS)

Uso de la información de los buzones de sugerencias

Limpieza de los servicios higiénicos

Existencia del buzón de sugerencias

Mejoras en el establecimiento a causa del uso del buzón

Referencias de los agentes comunitarios de salud a los

establecimientos

Seguimiento de pacientes con neumonía

Existencia de Radar de gestantes actualizado

Limpieza de la sala de espera

$\begin{array}{ccccccccccc}1 & 2 & 2 & 1 & 1 & 1 & 2 & 2 & 1 & 15 & 1,5 \\ 2 & 3 & 2 & 2 & 2 & 2 & 2 & 2 & 1 & 20 & 2,0 \\ 2 & 2 & 3 & 3 & 2 & 2 & 2 & 3 & 2 & 23 & 2,3 \\ 1 & 1 & 2 & 2 & 2 & 1 & 2 & 2 & 2 & 16 & 1,6 \\ 1 & 2 & 2 & 2 & 1 & 1 & 2 & 1 & 2 & 15 & 1,5 \\ & & & & & & & & & \end{array}$

Puntaje total para nivel calidad de atención

$\begin{array}{rrrrrrrrrrrr}3 & 2 & 1 & 3 & 3 & 2 & 2 & 2 & 2 & 2 & 22 & 2,2 \\ 1 & 2 & 2 & 3 & 3 & 2 & 1 & 2 & 3 & 2 & 21 & 2,1 \\ 1 & 1 & 3 & 1 & 2 & 2 & 2 & 2 & 1 & 2 & 17 & 1,7 \\ 1 & 1 & 2 & 1 & 2 & 1 & 1 & 2 & 2 & 1 & 14 & 1,4 \\ 2 & 2 & 2 & 2 & 3 & 2 & 2 & 3 & 3 & 2 & 23 & 2,3 \\ 3 & 3 & 2 & 3 & 3 & 2 & 3 & 3 & 3 & 3 & 28 & 2,8 \\ & & & & & & & & & & & \\ 2 & 3 & 2 & 2 & 3 & 3 & 3 & 3 & 3 & 3 & 27 & 2,7 \\ 2 & 2 & 2 & 2 & 2 & 3 & 3 & 3 & 2 & 2 & 23 & 2,3 \\ 1 & 1 & 2 & 1 & 2 & 2 & 2 & 2 & 2 & 2 & 17 & 1,7 \\ & & & & & & & & & & \text { Subtotal } & 19,2 \\ & & & & & & & & & & 101,62\end{array}$




\section{Referencias}

1. McCaw-Binns A, Standard-Goldson A, Ashley D, Walker G, MacGillivray I. Access to care and maternal mortality in Jamaican hospitals: 1993-1995. Int J Epidemiol 2001; 30: 796-801.

2. WHO (World Health Organization). Reduction of maternal mortality: a joint WHO/UNFPA/UNICEF/World Bank statement. Geneva: The Organization; 1999.

3. Starrs A. Preventing the tragedy of maternal deaths: a report on the international. In: The Safe Motherhood Agenda: priorities for the next decade: report on the Safe Motherhood Technical Consultation. Colombo; 1997.

4. OPS (Organización Panamericana de la Salud). Plan de Acción Regional para la Disminución de la Mortalidad Materna en las Américas. Washington (DC): La Organización; 1990.

5. Acosta A, Cabezas E, Chaparro J. Present and future of maternal mortality in Latin America. Int J Gynecol Obstet 2000; 70: $125-31$

6. OPS (Organización Panamericana de la Salud). La mortalidad materna en la Región Andina: una tragedia evitable. In: Informe de la Conferencia Andina sobre Maternidad sin Riesgos; 1993 feb 16-21; Santa Cruz de la Sierra, Bolivia. Santa Cruz de la Sierra: Family Care International; 1993.

7. McDonagh M. Is antenatal care effective in reducing maternal morbidity and mortality? Health Policy Plann 1996; 11: 115

8. Rooney C. Antenatal care and maternal health: how effective is it? A review of the evidence. Geneva: WHO, Division of Family Health: 1992.

9. Ross G, Zeballos J, Infante A. La calidad y la reforma del sector salud en América Latina y el Caribe. Rev Panam Salud Pública 2000; 8: 93-7.

10. Langer A, Nigenda G, Catino J. Health sector reform and reproductive health in Latin America and the Caribbean strengthening the links. Bull World Health Organ 2000; 78: 667-76.

11. Donnay F. Maternal survival in developing countring: what has been done, what can be achieved in the next decade. Int J Gynecol Obstet 2000; 70: 89-97.

12. Ronsmans C, Vanneste AM, Chakraborty J, van Ginneken J. Decline in maternal mortality in Matlab, Bangladesh: a cautionary tale. Lancet 1997; 350: 1810-4.

13. Habicht JP, Victora CG, Vaughan JP. Evaluation designs for adecuacy, plausibility and probability of public health programme performance and impact. Int J Epidemiol 1999; 28: $10-8$.

14. OPS (Organización Panamericana de la Salud). Evaluación del Plan de Acción Regional para la Reducción de la Mortalidad Materna 1990-1994. Washington (DC): La Organización; 1996.

15. Peru. Instituto Nacional de Estadística e Informática. Encuesta nacional de demografía y salud 1996: Lima: El Instituto; 1996.

16. Peru. Ministerio de Salud. Normas técnico administrativas para la atención integral de la salud materno perinatal. Lima: El Ministerio; 1995.

17. Peru. Ministerio de Salud. Lineamientos de Políticas del Sector Salud 1995-2000: hacia un sector salud con eficiencia equidad y calidad. Lima: El Ministerio; 1996.

18. Bruce J. Fundamental elements of the quality of care: a simple framework. Stud Fam Plann 1990; 21: 61-91.

19. Peru. Ministerio de Salud. Proyecto 2000: mejoramiento continuo de calidad de servicios de salud materno infantil. Lima: El Ministerio; 1998.
20. Peru. Ministerio de Salud. Estimaciones de población por grupos de edad según departamento, provincia y distrito. Lima: Oficina de Estadística e Informática; 2000.

21. Instituto Cuánto. Encuesta nacional de niveles y condiciones de vida 1997. Lima: El Instituto; 1997.

22. Riegleman R, Hirsch R. Cómo estudiar un estudio y probar una prueba. Washington (DC): OPS; 1992. (Publicación Científica, 531).

23. Acharya L, Cleland J. Maternal and child health services in rural Nepal: does access or quality matter more. Health Policy Plann 2000; 15: 223-9.

24. Parvez M. Utilization of rural basic health services in Pakistan: report of evaluation study. Alexandria: WHO; 1994.

25. Bloom S, Lippeveld T, Wypij D. Does antenatal care makes a difference to safe delivery? A study in urban Uttar Pradesh, India. Health Policy Plann 1999; 14: 38-48.

26. Kwast B, Liff J. Factors associated with maternal mortality in Addis Ababa, Ethiopia. Int J Epidemiol 1988; 17: 11521.

27. Anandalakshmy P, Talwar PP. Demographic, socio-economic and medical factors affecting maternal mortality - an Indian experience. J Fam Welfare 1993; 39: 1-4.

28. Mbizvo M, Fawcus S, Lindmark G, Nystrom L. Operational factors of maternal mortality in Zimbabwe. Health Policy Plann 1993; 8: 369-78.

29. Midhet F, Becker S, Berendes H. Contextual determinants of maternal mortality in rural Pakistan. Soc Sci Med 1998; 46: $1587-98$

30. Shiffman J. Can poor countries surmount high maternal mortality? Stud Fam Plann 2000; 31: 274-89.

31. Magadi M, Diamond I, Madise N. Analysis of factors associated with maternal mortality in Kenyan hospitals. J Biosoc Sci 2001; 33: 375-89.

32. Maine D, Rosenfield A. The safe motherhood initiative: why has it stalled? Am J Public Health 1999; 89: 480-2.

33. Le Bacq F, Rietsema A. High maternal mortality levels and additional risk from poor accesibility in two districts of Northern province, Zambia. Int J Epidemiol 1997; 25: 357-63.

34. Walraven G, Telfer M, Rowley J, Ronsmans C. Maternal mortality in rural Gambia: level, causes and contributing factors. Bull World Health Organ 2000; 78: 603-13.

35. Makhlouf C, Potter J. Maternal health care utilization in Jordan: a study of patterns and determinants. Stud Fam Plann 1991; 22: 177-87.

36. Cervantes R, Watanabe T, Denegri J. Mortalidad materna y mortalidad perinatal en hospitales del Perú. Lima: Sociedad Peruana de Ginecología; 1988.

37. Perú. Ministerio de Salud. Evaluación del Programa Nacional de Salud Materno Perinatal 2000. Lima: El Ministerio; 2000.

38. Thadeus S, Maine D. Too far to walk: maternal mortality in context. Soc Sci Med 1998; 38: 1091-110.

39. Abou ZC, Royston E. Maternal mortality: a global factbook. Geneva: WHO; 1991. (WHO/MSH/MSM/91.3).

40. Prual A, Toure A, Huguet D, Laurent Y. The quality of risk factor screening during antenatal consultations in Niger. Health Policy Plann 2000; 15: 11-6.

41. Hotchkiss D. Expansion of rural health care and the use of maternal services in Nepal. Health Place 2001; 7: 39-45.

42. Weil O, Fernandez H. Is safe motherhood an orphan initiative? Lancet 1999; 354: 940-3.

43. Fauveau V, Steward K, Khan S. Effect on mortality of community-based maternity-care programme in rural Bangladesh. Lancet 1991; 338: 1183-6.

Recebido em 8 de agosto de 2003

Versão final reapresentada em 31 de outubro de 2003

Aprovado em 10 de novembro de 2003 